\title{
Improving frailty identification and comprehensive geriatric assessment (CGA) completion on the wards
}

\author{
Authors: Divya Verma, ${ }^{\mathrm{A}}$ Myuran Kaneshamoorthy, ${ }^{\mathrm{A}}$ Leila Bafadhel, ${ }^{\mathrm{A}}$ Francesca Bonora ${ }^{\mathrm{A}}$ and Rebecca Walker ${ }^{\mathrm{A}}$
}

\section{Introduction}

Comprehensive geriatric assessment (CGA) is known to deliver substantial and measurable health improvements to frail older people, including increased independence and a reduction in mortality. ${ }^{1}$ The Clinical Frailty Scale (CFS) can detect older adults at higher risk of complicated course and longer hospital stay. ${ }^{2}$ Despite the known benefits, previous audits have shown poor documentation on geriatric wards at Southend Hospital. Therefore, we devised a quality improvement project to improve the uptake of both these.

\section{Method}

A total of two plan, do, study, act (PDSA) cycles were completed where CGA completion and CFS documentation were audited. Each cycle lasted 2 weeks ( 25 patients). Qualitative feedback was obtained from the members of the multidisciplinary team to aid improvements. The baseline audit was based on the introduction of a two-page ward pro forma for all new patients. The first intervention was an improved two-page ward pro forma. The second intervention was a one-page ward pro forma.

\section{Results}

Originally, $40 \%$ of new patients admitted onto the ward had a CGA and CFS score. After the first intervention, 79\% (19) patients had a CFS score and a CGA; $21 \%$ had a full CGA completed and $58 \%$ had partial CGA. Feedback included wanting a single page pro forma to increase uptake. Questions needed to be more unambiguous and more tick boxes. After the second intervention 100\% (25) patients had a CFS score and a CGA; $40 \%$ (10) had a full CGA completed and $60 \%$ (15) had a partial CGA. Feedback include incorporating the ward round documentation to avoid repetition.

\section{Conclusions}

The results show that by using a focused, concise and user-friendly pro forma, uptake of GCA and CFS can be significantly increased, bringing substantial and measurable health improvements to frail older people admitted to elderly care wards.

Authors: ASouthend University Hospital NHS Foundation Trust, Southend, UK

\section{Conflicts of interest}

None declared.

\section{References}

1 Welsh T], Gordon AL, Gladman JR. Comprehensive geriatric assessment-a guide for the non-specialist. Int J Clin Pract 2014;68:290-3.

2 Juma S, Taabazuing MM, Montero-Odasso M. Clinical Frailty Scale in an acute medicine unit: a simple tool that predicts length of stay. Can Geriatr J 2016;19:34-9. 